\title{
EFFECT OF A ONE-SESSION-PER-WEEK PHYSICAL EDUCATION-BASED STRETCHING PROGRAM ON HAMSTRING EXTENSIBILITY IN SCHOOLCHILDREN
}

\author{
Daniel Mayorga-Vega ${ }^{1}$, Rafael Merino-Marban ${ }^{2}$, \\ Francisco Javier Redondo-Martín ${ }^{3}$, and Jesús Viciana ${ }^{1}$ \\ ${ }^{1}$ Department of Physical Education and Sport, University of Granada, Spain \\ ${ }^{2}$ Facultad de Ciencias de la Educación, Universidad de Málaga (UMA), Spain \\ ${ }^{3}$ CEIP Manuel Laza Palacios, Junta de Andalucía, Spain
}

Original scientific paper

UDC: $371.3: 796.4-053.5$

\begin{abstract}
:
The purpose of this study was to examine the effects of a one-session-per-week stretching program on hamstring extensibility among schoolchildren in the physical education (PE) setting. Thirty seven 9-year-old schoolchildren from two classes were clustered and randomly assigned to an experimental group ( $\mathrm{n}=19)$ or a control group $(\mathrm{n}=18)$. During PE classes, the experimental students performed a 3-minute stretching program once a week throughout academic year (a total of 32 calendar weeks, but 28 weeks of intervention after excluding holidays). Hamstring extensibility (estimated by the classic sit-and-reach test) was assessed at the beginning (week 0), in the middle (week 18) and at the end (week 34) of the stretching intervention program. The results of the two-way analysis of variance showed that the PE-based stretching program improved statistically significantly the students' sit-and-reach scores in the middle and at the end of the intervention $(p<.01)$. Since in PE many curricular contents need to be developed each academic year and the subject is also restricted by its limited curriculum time allocation, teachers could improve students' hamstring extensibility by only a one-session-per-week stretching program. Therefore, in addition to the improvement of students' flexibility levels, this intervention program might permit regular development of other PE curricular contents. This knowledge could help and guide teachers to design programs that guarantee a feasible and effective development of flexibility in the PE setting.
\end{abstract}

Key words: flexibility program, frequency, classic sit-and-reach test, health-related physical fitness, primary school, physical education setting

\section{Introduction}

Physical fitness is nowadays considered as one of the most important health markers in childhood (Ortega, Ruiz, Castillo, \& Sjöström, 2008) and flexibility is an important component of health-related physical fitness (Centers for Disease Control and Prevention, 2011). Although flexibility has been traditionally referred to as the maximum range of motion in a joint or series of joints, recently it has been recommended that it should be expressed as muscle extensibility (Alter, 2004), that is, the ability of a muscle to be elongated or stretched (Clark, Sutton, \& Lucett, 2013). Particularly, children with an adequate hamstring extensibility seem to have a lower risk of pelvic/spinal postural disorders (de Lemos, dos Santos \& Gaya, 2012; López-Miñarro \& Alacid, 2010; Muyor, Alacid, Rodríguez-García, \&
López-Miñarro, 2012), current low back pain (Jones, Stratton, Reilly, \& Unnithan, 2005; Roy, Shaw, \& Beattie, 2015) and neck tension (Mikkelsson, et al., 2006), as well as a lower risk of low back pain later during adulthood (Hestbaek, Leboeuf-Yde, Kyvik, \& Manniche, 2006). Additionally, in children with low back pain an improvement in their hamstring extensibility levels has also been associated to a reduction of their pain levels (Ahlqwist, Hagman, Kjellby-Wendt, \& Beckung, 2008; Jones, Stratton, Reilly, \& Unnithan, 2007).

Therefore, health promotion policies should be designed to identify children with low health-related physical fitness levels, as well as to encourage them to achieve a health-enhancing physical activity levels (Ortega, et al., 2008). In many circumstances, schools may play an important role in this public health issue (Ortega, et al., 2008). Particularly, a 
shortened hamstring could be addressed proactively through the subject of physical education (PE) by a systematic performance of stretching exercises (Santonja, Sainz De Baranda, Rodríguez, López, \& Canteras, 2007; Thacker, Gilchrist, Stroup, \& Kimsey, 2004). In this line, PE teachers in most countries are nowadays required to develop and maintain students' health-enhancing flexibility levels (European Commission/EACEA/Eurydice, 2013).

Previous studies have found that a PE-based stretching program, carried out twice a week, improves hamstring extensibility in schoolchildren (e.g. Kamandulis, Emeljanovas, \& Skurvydas, 2013; Merino-Marban, Mayorga-Vega, Fernandez-Rodriguez, Vera Estrada, \& Viciana, 2015). However, nowadays PE teachers must face several planningrelated problems for developing students' flexibility levels (Viciana, Mayorga-Vega, \& MerinoMarban, 2014). For instance, apart from the fact that many curricular contents must be developed each academic year, PE is usually restricted by its limited curriculum time allocation. Moreover, this restriction is especially accented when the number of PE sessions a week is limited to only two, which is the norm in most European countries (European Commission/EACEA/Eurydice, 2013).

Since stretching programs cannot be allocated a large part of PE time, the application of a onesession-per-week stretching program could be more suitable. Unfortunately, to our knowledge there are no studies examining the effect of a one-sessionper-week stretching program in schoolchildren and related studies with adults are really scarce (Marques, Vasconcelos, Cabral, \& Sacco, 2009). Currently, there is a lack of scientific information about the effects of this kind of programs among children and, therefore, research in this area is required. Consequently, the purpose of the present study was to examine the effects of a one-sessionper-week stretching program on hamstring extensibility among schoolchildren in the PE setting.

\section{Methods}

\section{Participants}

The study protocol was first approved by the Ethical Committee of the University of Malaga. After the school approvals had been obtained, children and their legal guardians were fully informed about all the features of the study and were required to sign an informed consent document. All 37 schoolchildren, 18 boys and 19 girls, from two different third-grade PE classes of a public primary school were invited and agreed to participate in the present study. For practical reasons and the nature of the present study (i.e. intervention focused on natural groups in a school setting) a cluster-randomized controlled design was used
(Mayorga-Vega, Viciana, \& Cocca, 2013; MerinoMarban, Mayorga-Vega, Fernandez-Rodriguez, Vera Estrada, \& Viciana, 2015). Natural classes were assigned randomly to either the control (CG) or experimental study group (EG).

All the participants were free of orthopedic disorders such as episodes of hamstring and/or lumbar injuries, fractures, surgery or pain in the spine or hamstring and/or lumbar muscles over the past six months (López-Miñarro, Sainz de Baranda, \& Rodríguez-Garcia, 2009). The inclusion criteria were: (a) correct performance of all the flexibility evaluations, and (b) attendance rate of $90 \%$ or higher at PE classes during the intervention period. All the participants met the above-mentioned inclusion criteria.

\section{Measures}

All the measures were performed during the regularly scheduled PE classes. Prior to the intervention, information about participants' gender, age and extra-curricular sport participation was collected. Then, anthropometric measures were also taken during the same class. Afterward, the flexibility test was applied at the beginning (pretest, week 0), in the middle (posttest, week 18) and at the end (retest, week 34) of the stretching intervention program. A week before of the baseline flexibility measure, a familiarization session was also carried out for all participants.

Extra-curricular sport. Data on the students' extra-curricular sport participation was collected by a short survey. Participants were asked if they were enrolled in any organized sport during their free time, which kind of sport(s), if they were federated, and how many days and average time per session they used to participate. Afterward, based on previous studies (Mayorga-Vega, Merino-Marban, Vera-Estrada, \& Viciana, 2014b; Merino-Marban, et al., 2015), data were dichotomized as schoolchildren that either regularly participated or did not in extra-curricular sport activities at least twice per week with an average session time equal or higher than 60 minutes.

Anthropometric measures. Participants' body mass and height were measured and then the body mass index (BMI) was calculated as body mass/ height squared $\left(\mathrm{kg} / \mathrm{m}^{2}\right)$. During the measurement of body mass and height participants were in shorts and T-shirts and barefoot. For the body mass measure, once the scale was reading zero, the participant stood in the centre of the scale without support and with the weight distributed evenly on both feet. For the body height measurement, the student stood with the feet together and the heels, buttocks and upper part of the back touching the scale, and with the head placed in the Frankfort plane. The average of two measurements for both body weight and height was retained (ISAK, 2001). 
Hamstring extensibility. Participants' hamstring extensibility was estimated by the classic sit-andreach (SR) test (Mayorga-Vega, Merino-Marban, \& Viciana, 2014c; Mayorga-Vega, Viciana, Cocca, \& Merino-Marban, 2014d). The SR test was applied by the same tester and instrument, a wooden box with a ruler on top (the score $23 \mathrm{~cm}$ corresponded to the tangent of the feet; accuracy $0.1 \mathrm{~cm}$ ). Additionally, the measures were performed in an indoor sports facility under similar environmental conditions, on the same day of the week and at the same time for each participant. Children were also reminded to avoid any exhausting physical activity $48 \mathrm{~h}$ prior to each evaluation session.

Prior to the evaluation, the participants performed a standardized warm-up consisting of three minutes of jogging at low intensity followed by two static and bipodal hamstring stretching exercises (two 15-second sets of each exercise). Then, the participants, in their sportswear and barefoot, were assessed by the SR test. Briefly, at the beginning of the test the participants stood in front of the box, then sat down with their hips flexed, knees extended and both hands on the top of the ruler. From this position, the participants had to bend the trunk forward slowly and progressively (no swings) in order to reach the furthest possible distance and to remain still for at least two seconds. Two trials were performed one minute apart, and the average was retained (Mayorga-Vega, Merino-Marban, \& Garcia-Romero, 2015).

\section{Procedures}

All the participants were enrolled in their standard PE classes. Additionally, the EG participants performed a stretching-based intervention program once a week for the whole academic year (a total of 32 calendar weeks, but 28 weeks of intervention after excluding holidays). If some EG participant missed an intervention session, he/she was allowed to catch up in the next PE session of the same week. On the other hand, the CG participants followed the same standard PE classes without performing any stretching exercise. The intervention program was conducted and supervised by the same PE teacher. Additionally, the participants were urged to maintain their normal physical activity levels outside of the supervised setting during the research period.

During each intervention session, the EG participants performed static hamstring stretches for three minutes during the cool-down period (Mayorga-Vega, Merino-Marban, Garrido, \& Viciana, 2014a). In the PE setting, this kind of intervention has been called "intermittent teaching unit" (Viciana \& Mayorga-Vega, 2016). Each intervention session included three 20 -second sets of three bipodal stretching exercises. Overall, four different stretching exercises were designed and alternated during the intervention program: (a) standing with feet together; (b) sitting with feet together; (c) standing with feet shoulders width apart, and (d) sitting with feet shoulders width apart (see Figure 1 in Merino-Marban, et al., 2015). Besides the fact that these exercises mainly stretched hamstring muscles, which was the objective of the present stretching intervention program, these drills were selected because they are very common and known to schoolchildren, they are safe and easy to perform at this age and less time consuming than unipodal exercises.

During the stretching exercises, the children flexed forward their bodies at the hip, trying to maintain the spine in neutral position as much as possible until a gentle stretch was felt in the hamstrings. The knees were fully extended and toes pointed to the ceiling with no hip rotation. The stretched positions were held gently until the end point of the range was reached (i.e. stretch to the point at which tightness of the hamstring muscles, but no pain was felt). Once this position was achieved, the children held it for 20 seconds.

\section{Statistical analyses}

Descriptive statistics (means and standard deviations) for body mass, body height, BMI, and baseline SR scores were calculated. Besides some common exploratory analyses for detecting potential data errors such as the extreme cases, statistical tests assumptions (i.e. normality, homogeneity of variance, etc.) were examined and met for all the tests conducted. A one-way analysis of variance (ANOVA) was used to study the differences of general characteristics and baseline SR scores between the groups. Moreover, chi-squared analyses were carried out to test the ratio differences of gender and extracurricular sport practitioners between the two groups. The test-retest reliability of the familiarization and pretest SR scores was estimated using the intraclass correlation coefficient from the two-way ANOVA (Shrout \& Fleiss, 1979), as well as the 95\% confidence interval. Afterward, the effect of the intervention program on hamstring extensibility was examined using a two-way ANOVA applied over the SR scores, including group as an independent variable (CG, $\mathrm{EG}$ ) and time as a dependent variable (pretest, posttest, retest). Subsequently, for the post hoc analyses, $\alpha$ values were corrected using the Bonferroni adjustment. Then, the Hedges' g effect size was used to examine the magnitude of intervention effects (Hedges, 2007). Effect size was interpreted as trivial ( $\mathrm{g}=.00-.19)$, small ( $\mathrm{g}=.20-.49)$, medium ( $\mathrm{g}=.50-.79)$, and large ( $\mathrm{g} \geq .80)$ (Cohen, 1992). All statistical analyses were performed using the SPSS version 20.0 for Windows (IBM ${ }^{\circledR}$ SPSS ${ }^{\circledR}$ Statistics 20). The statistical significance level was set at $\mathrm{p}<.05$. 
Table 1. General characteristics (mean \pm standard deviation/frequency) of the participants and differences between experimental and control groups

\begin{tabular}{lccccc}
\hline & \multirow{2}{*}{$\begin{array}{c}\text { Sample } \\
(\mathrm{N}=37)\end{array}$} & $\begin{array}{c}\text { Experimental } \\
(\mathrm{n}=19)\end{array}$ & $\begin{array}{c}\text { Control } \\
(\mathrm{n}=18)\end{array}$ & \multicolumn{2}{c}{ Differences $^{\mathrm{a}}$} \\
\cline { 5 - 7 } & $36.45 \pm 8.03$ & $35.43 \pm 5.89$ & $37.53 \pm 9.87$ & $\mathrm{~F} / \chi^{2}$ & $\mathrm{p}$ \\
\hline Body mass $(\mathrm{kg})$ & $1.36 \pm .06$ & $1.35 \pm .04$ & $1.37 \pm .07$ & .734 & .397 \\
Body height $(\mathrm{m})$ & $19.63 \pm 3.52$ & $19.41 \pm 3.20$ & $19.86 \pm 3.92$ & .142 & .708 \\
Body mass index $\left(\mathrm{kg} / \mathrm{m}^{2}\right)$ & $18 / 19$ & $8 / 11$ & $10 / 8$ & .669 & .413 \\
Gender $($ boys $/$ girls) & $17 / 20$ & $11 / 8$ & $6 / 12$ & 2.245 & .134 \\
Extra-curricular sport (yes $/ \mathrm{no})^{\mathrm{b}}$ & $24.09 \pm 6.28$ & $24.01 \pm 5.53$ & $24.18 \pm 7.16$ & .007 & .933 \\
Sit-and-reach pretest score $(\mathrm{cm})$ & & & &
\end{tabular}

Note. ${ }^{a}$ Significance level from the one-way ANOVA for the body mass, body height, body mass index, and sit-and-reach pretest score, and from the chi-squared test for the gender and extra-curricular sport ratios. ${ }^{b}$ Children that regularly participated (yes) or did not (no) at least twice per week in extra-curricular sport activities.

Table 2. Effect of the stretching-based intervention program on sit-and-reach scores $(\mathrm{cm})$

\begin{tabular}{|c|c|c|c|c|c|c|c|}
\hline \multirow{2}{*}{ Group } & \multirow{2}{*}{$\begin{array}{c}\text { Pretest (1) } \\
(\mathrm{M} \pm \mathrm{SD})\end{array}$} & \multirow{2}{*}{$\begin{array}{l}\text { Posttest (2) } \\
(\mathrm{M} \pm \mathrm{SD})\end{array}$} & \multirow{2}{*}{$\begin{array}{c}\text { Retest (3) } \\
(\mathrm{M} \pm \mathrm{SD})\end{array}$} & \multirow{2}{*}{$p^{a}$} & \multicolumn{3}{|c|}{ Effect size $^{b}$} \\
\hline & & & & & $1-2$ & $2-3$ & $1-3$ \\
\hline Experimental $(n=19)$ & $24.0 \pm 5.5$ & $25.5 \pm 5.8^{*}$ & $27.6 \pm 4.6^{\wedge} \dagger$ & \multirow{2}{*}{$<.001$} & \multirow{2}{*}{.28} & \multirow{2}{*}{.30} & \multirow{2}{*}{.60} \\
\hline Control $(n=18)$ & $24.2 \pm 7.2$ & $23.9 \pm 7.7$ & $24.0 \pm 7.7$ & & & & \\
\hline
\end{tabular}

Note. $\mathrm{M}=$ mean; $\mathrm{SD}=$ standard deviation. aSignificance level from the two-way ANOVA with the post-hoc analysis with Bonferroni adjustment: the change statistically significant from pretest to posttest $\left({ }^{*} p<.01\right)$, from posttest to retest $\left({ }^{\wedge} p<.001\right)$, and from pretest

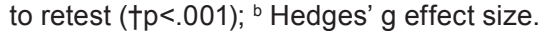

\section{Results}

General characteristics of the participants studied are shown in Table 1. The one-way ANOVA results did not show statistically significant differences in body mass, body height, BMI, or SR baseline values between EG and CG ( $p>.05)$. Furthermore, the chi-square analyses showed that the two groups had a balanced representation of boys and girls and extra-curricular sport practitioners and non-practitioners ( $p>05)$. The test-retest reliability for SR scores was .98 (.95-.99).

Table 2 shows the effect of the stretching intervention program on SR scores. The results of the two-way ANOVA on the average obtained in the SR showed interaction effects between the group and time variables $\left[\mathrm{F}(2,70)=16.233 ; \mathrm{p}<.001 ; \eta^{2}=.317\right.$; $\mathrm{P}=.998$ ]. Subsequently, for post hoc analysis, the ANOVA with the Bonferroni adjustment showed that the EG improved hamstring extensibility statistically significantly from pretest to posttest $(p=.009)$, from posttest to retest $(p<.001)$, and from pretest to retest $(p<.001)$. On the other hand, for the CG no significant differences were found $(\mathrm{p}=1.000)$.

\section{Discussion and conclusions}

The purpose of the present study was to examine the effects of a one-session-per-week stretching program on hamstring extensibility among schoolchildren in the PE setting. The results of the current study showed that the PE-based stretching program performed only once a week improves hamstring extensibility in schoolchildren. Similar to the present study, Marques et al. (2009) found that the stretching program carried out once a week also improved adults' hamstring extensibility. However, we have to be conscious about these results, given the fact that the population examined were adults instead of children and that the study did not follow a controlled design.

Regarding the magnitude effects of the intervention, at the end of the academic year the effect size of the present study was medium indicating that the stretching program was effective. In contrast with the current results, all the previous studies carried out twice a week for the whole school year (31-32 weeks) obtained large and very large effect sizes ( $\mathrm{g}=.85-2.06$ ) (Rodríguez, Santonja, LópezMiñarro, Sáinz de Baranda, \& Yuste, 2008; Sainz de Baranda, 2009; Sainz de Baranda, et al., 2006; Santonja, et al., 2007). Apart from other training factors, such as the time of stretching per session (i.e. in all the above-mentioned studies it was 5-7 minutes comparing to 3 minutes in the present study), week frequency might clearly influence the magnitude effect of the intervention (i.e. two sessions per week instead of once a week in the present study). In this line, Santonja et al. (2007) found that when children performed stretching drills four times per week instead of two, the magnitude effect doubled (twice a week, $g=.85$; four times a week, $\mathrm{g}=1.53$; both 5 minutes per session, 31 weeks of duration). 
The frequency of the stretching program could have a positive relationship with the effect size of the intervention. However, since in most European countries PE is limited to only two sessions a week (European Commission/EACEA/Eurydice, 2013), the application of stretching programs with a higher frequency is not feasible in this setting. In this line, Merino-Marban et al. (2015) indicated that the increase of active time for learning in extra-curricular periods would represent an excellent strategy for PE teachers to pursue important objectives such as the flexibility improvement. Nevertheless, since this strategy mainly depends on students' autonomy, we have to be aware that in primary schoolchildren it could be impractical (Merino-Marban, et al., 2015). Therefore, although the frequency of the stretching programs seems to show a positive relationship with the effect size of the intervention, even a PE-based stretching program performed only once a week is effective among schoolchildren.

On the other hand, the duration of the intervention program also seems to be an important training factor. While the previous studies examining the effects of long-term stretching programs (a whole school year, 31-32 weeks) obtained large and very large effect sizes ( $\mathrm{g}=.85-2.06)$ (Rodríguez, et al., 2008; Sainz de Baranda, 2009; Sainz de Baranda, et al., 2006; Santonja, et al., 2007), the magnitude was lower for the mid-term stretching programs (16 weeks) ( $\mathrm{g}=.85-.88)$ (Coledam, Arruda, \& Ramos de Oliveira, 2012), and even lower for those with shortterm programs (8-10 weeks) $(\mathrm{g}=.35-.67)$ (MayorgaVega, et al., 2014a, 2014b; Merino-Marban, et al., 2015; Sánchez Rivas, Mayorga-Vega, Fernández Rodríguez, \& Merino-Marban, 2014). In this line, in the present study the effect size obtained in the midterm (after 17 weeks of intervention) was about the half that of the whole academic year (after 32 weeks of intervention). Although an effect size equal to .28 is generally interpreted as small (Cohen, 1992), we have to be aware that in education research even these values could be considered of practical significance (Valentine \& Cooper, 2003).

The main limitation of the present study was related to the validity of the test used. Muscle extensibility is commonly operationalized by the maximum range of motion in a joint or series of joints (Alter, 2004). Particularly, the straight leg raise test, which specifically measures the angular hip flexion, has been widely considered the criterion measure or "gold standard" of hamstring extensibility (Mayorga-Vega, et al., 2014c). Nevertheless, as in the present study, when the use of this angular test is limited because of practical issues such as time constraints, a recent meta-analysis about the criterion-related validity of the SR tests concluded that the classic SR test has an adequate validity for estimating hamstring extensibility among children
(Mayorga-Vega, et al., 2014c).

There are also some potential factors that could affect the validity of the classic SR test. A potential factor that might affect the validity of the classic SR test is the difference in children's length proportion between the upper and lower limbs (Hoeger, Hopkins, Button, \& Palmer, 1990). Nevertheless, since in the current study the pretest values were assessed, any change in the children's hamstring extensibility was compared with that baseline levels. In this way, in the present study children's length proportion between the upper and lower limbs could not affect the results of the study (Merino-Marban, et al., 2015). Another potential factor that could affect the validity of the classic SR test is the change in the proportion of the anthropometric parameters and/or in the position of the spine between the pretest and the post/retest measures. However, the fact that the study design included an equivalent $\mathrm{CG}$ (i.e. characteristics such as age, gender ratio, body mass, body height, BMI, extracurricular sport participation, and SR pretest scores were equivalent between both groups) must be highlighted. Thus, it could reasonably be assumed that any change in the proportion of the anthropometric parameters and/or in the position of the spine should be similar in both EG and CG (Merino-Marban, et al., 2015). Anyway, future research studies should examine the effect of long-term PE-based stretching programs performed once a week measured by an angular test.

On the other hand, another potential limitation of the present study could be related to the change of temperature in the different flexibility evaluation sessions. Nevertheless, since in the current study some measures were taken into account, we can be reasonably sure that this issue did not affect the cause-effect relationship. Firstly, Malaga (located in the South of Spain) is one of the warmest and most temperature-stable cities in Europe. Secondly, besides the fact that evaluations were conducted in an indoor sports facility that provided reasonably stable temperature environment, they were executed in autumn and at the beginning of spring and summer, when the temperature conditions in the city are similar. Furthermore, because of the fact that participants performed a standardized warm-up prior to the evaluations, the examined schoolchildren had an adequate muscular temperature to perform at their maximum during each flexibility evaluation. Finally, it must also be highlighted that the change pre-post intervention (post-retest/preretest alike) in the EG was compared with an equivalent $\mathrm{CG}$ that performed each evaluation session under the same environmental conditions. Therefore, any potential extraneous variable that could affect the intervention outcomes, such as the change of environmental temperature, was controlled by the CG. 
In conclusion, the present study is, to our knowledge, the first one that examines the effect of a one-session-per-week stretching program on hamstring extensibility among schoolchildren in the PE setting. The results of this study suggest that it is possible to improve students' hamstring extensibility by only one 3-minute session per week in the PE setting (i.e. only $3 \%$ of the PE time in a common situation of 50-minute sessions twice a week). Therefore, in addition to the improvement of students' hamstring extensibility levels, this intervention program might permit regular development of other PE curricular contents.

Unlike other physical fitness components, such as cardiorespiratory fitness where exercise produces overall benefits, flexibility is characteristic because exercise has an effect only on the specific muscle that is stretched (Alter, 2004). Therefore, despite the fact that hamstring extensibility improvement is a key region in the health-related flexibility promotion (Jones, et al., 2005; Mikkelsson, et al., 2006), PE teachers should actually develop and maintain students' health-enhancing levels of the whole body flexibility. Unfortunately, the main practical limitation on that goal is the fact that it would eat up too much available time in PE planning (Viciana, et al., 2014). Therefore, in order to improve the whole body flexibility in schoolchildren, some innovative strategies should be designed instead. In this line, Merino-Marban et al. (2015) indicated that the increase of active time for learning in extra-curricular periods would represent an excellent strategy for PE teachers to pursue the whole body flexibility improvement. Besides improving key body regions, such as hamstring extensibility during PE sessions, teachers should also teach their students how to properly develop and maintain the whole body flexibility during their free time. Nevertheless, since this strategy mainly depends on the students' autonomy, we have to be aware that in primary schoolchildren the results could be limited. Future research studies should examine the effect of innovative strategies that allow schoolchildren to achieve and maintain health-enhancing levels of the whole body flexibility during their free time. Furthermore, the effect of long-term PE-based stretching programs with different weekly frequencies should also be examined. Finally, future research studies should contrast the present results with a wider age sample including schoolchildren from primary to secondary education.

\section{References}

Ahlqwist, A., Hagman, M., Kjellby-Wendt, G., \& Beckung, E. (2008). Physical therapy treatment of back complaints on children and adolescents. Spine, 33(20), e721-e727.

Alter, M.J. (2004). Science of flexibility ( $3^{\text {rd }}$ ed.). Champaign, IL: Human Kinetics.

Centers for Disease Control and Prevention. (2011). School health guidelines to promote healthy eating and physical activity. Retrieved from http://www.cdc.gov/healthyschools/npao/pdf/mmwr-school-health-guidelines.pdf on March of 2015.

Clark, M.A., Sutton, B.G., \& Lucett, S.C. (Eds.). (2013). NASM essentials of personal fitness training (4th ed.). Baltimore, MD: Lippincott Williams \& Wilkins.

Cohen, J.A. (1992). Power primer. Psychological Bulletin, 112(1), 155-159.

Coledam, D.H.C., Arruda, G.A., \& Ramos de Oliveira, A. (2012). Chronic effect of static stretching performed during warm-up on flexibility in children. Revista Brasileira de Cineantropometria e Desempenho Humano, 14(3), 296-304.

De Lemos, A.T., dos Santos, F.R., \& Gaya, A.G. (2012). Lumbar hyperlordosis in children and adolescents at a privative school in southern Brazil: Occurrence and associated factors. Cadernos de Saúde Pública, 28(4), 781-788.

European Commission/EACEA/Eurydice. (2013). Physical education and sport at school in Europe Eurydice Report. Luxembourg: Publications Office of the European Union.

Hedges, L.V. (2007). Effect sizes in cluster-randomized designs. Journal of Educational and Behavioral Statistics, $32(4), 341-370$.

Hestbaek, L., Leboeuf-Yde, C., Kyvik, K.O., \& Manniche, C. (2006). The course of low back pain from adolescence to adulthood. Eight year follow up of 9600 twins. Spine, 31(4), 468-472.

Hoeger, W.W., Hopkins, D.R., Button, S., \& Palmer, T.A. (1990). Comparing the sit and reach with the modified sit and reach in measuring flexibility in adolescents. Pediatric Exercise Science, 2(2), 156-162.

International Society for the Advancement of Kinanthropometry (ISAK). (2001). International Standards of Anthropometric Assessment. Australia: ISAK. 
Jones, M.A., Stratton, G., Reilly, T., \& Unnithan, V.B. (2005). Biological risk indicators for recurrent non-specific low back pain in adolescents. British Journal of Sports Medicine, 39(3), 137-140.

Jones, M., Stratton, G., Reilly, T., \& Unnithan, V. (2007). The efficacy of exercise as an intervention to treat recurrent nonspecific low back pain in adolescents. Pediatric Exercise Science, 19(3), 349-359.

Kamandulis, S., Emeljanovas, A., \& Skurvydas, A. (2013). Stretching exercise volume for flexibility enhancement in secondary school children. Journal of Sports Medicine and Physical Fitness, 53(6), 687-692.

López-Miñarro, P.A., \& Alacid, F. (2010). Influence of hamstring muscle extensibility on spinal curvatures in young athletes. Science and Sports, 25(4), 188-193.

López-Miñarro, P.A., Sainz de Baranda, P., \& Rodríguez-Garcia, P.L. (2009). A comparison of the sit-and-reach test and the back-saver sit-and-reach test in university students. Journal of Sports Science and Medicine, 8(1), 116-122.

Marques, A.P., Vasconcelos, A.A., Cabral, C.M.N., \& Sacco, I.C. (2009). Effect of frequency of static stretching on flexibility, hamstring tightness and electromyographic activity. Brazilian Journal of Medical and Biological Research, 42(10), 949-953.

Mayorga-Vega, D., Merino-Marban, R., \& Garcia-Romero, J.C. (2015). Validity of sit-and-reach with plantar flexion test in children aged 10-12 years. Revista Internacional de Medicina y Ciencias de la Actividad Física y del Deporte, 15(59), 577-591.

Mayorga-Vega, D., Merino-Marban, R., Garrido, F.J., \& Viciana, J. (2014a). Comparison between warm-up and cooldown stretching programs on hamstring extensibility gains in primary schoolchildren. Physical Activity Review, $2,16-24$.

Mayorga-Vega, D., Merino-Marban, R., Vera-Estrada, F., \& Viciana, J. (2014b). Effect of a short-term physical education-based flexibility program on hamstring and lumbar extensibility and its posterior reduction in primary schoolchildren. Kinesiology, 46(2), 227-233.

Mayorga-Vega, D., Merino-Marban, R., \& Viciana, J. (2014c). Criterion-related validity of sit-and-reach tests for estimating hamstring and lumbar extensibility: A meta-analysis. Journal of Sports Science and Medicine, 13(1), 1-14.

Mayorga-Vega, D., Viciana, J., \& Cocca, A. (2013). Effects of a circuit training program on muscular and cardiovascular endurance and their maintenance in schoolchildren. Journal of Human Kinetics, 37, 153-160.

Mayorga-Vega, D., Viciana, J., Cocca, A., \& Merino-Marban, R. (2014d). Criterion-related validity of toe-touch test for estimating hamstring extensibility: A meta-analysis. Journal of Human Sport and Exercise, 9(1), 188-200.

Merino-Marban, R., Mayorga-Vega, D., Fernandez-Rodriguez, E., Vera Estrada, F., \& Viciana, J. (2015). Effect of a physical education-based stretching programme on sit-and-reach score and its posterior reduction in elementary schoolchildren. European Physical Education Review, 21(1), 83-92.

Mikkelsson, L., Nupponen, H., Kaprio, J., Kautiainen, H., Mikkelsson, M., \& Kujala, U. (2006). Adolescent flexibility, endurance strength, and physical activity as predictors of adult tension neck, low back pain, and knee injury: A 25 year follow up study. British Journal of Sports Medicine, 40(2), 107-113.

Muyor, J.M., Alacid, F., Rodríguez-García, P.L., \& López-Miñarro, P.A. (2012). Influence of hamstring extensibility on sagittal spinal curvatures and pelvic inclination in athletes. International Journal of Morphology, 30(1), 176-181.

Ortega, F.B., Ruiz, J.R., Castillo, M.J., \& Sjöström, M. (2008). Physical fitness in childhood and adolescence: A powerful marker of health. International Journal of Obesity, 32(1), 1-11.

Rodríguez, P.L., Santonja, F.M., López-Miñarro, P.A., Sáinz de Baranda, P., \& Yuste, J.L. (2008). Effect of physical education stretching program on sit-and-reach score in schoolchildren. Science and Sports, 23(3-4), 170-175.

Roy, S.L., Shaw, P.C., \& Beattie, T.F. (2015). Low back pain in the paediatric athlete. European Journal of Emergency Medicine, 22(5), 348-354.

Sainz de Baranda, P. (2009). Flexibility training in physical education: Intervention program. Cultura, Ciencia y Deporte, 4(10), 33-38.

Sainz de Baranda, P., Rodríguez, P.L., Santonja, F.M., López, P.A., Andújar, P., Ferrer V., \& Pastor, A. (2006). Effects of hamstring stretching exercises on the toe-touch test in elementary schoolchildren. Journal of Human Movement Studies, 51(4), 277-289.

Sánchez Rivas, E., Mayorga-Vega, D., Fernández Rodríguez, E., \& Merino-Marban, R. (2014). Effect of a hamstring stretching program during physical education lessons in primary education. Journal of Sport and Health Research, 6(2), 159-168.

Santonja, F.M., Sainz De Baranda, P., Rodríguez, P.L., López, P.A., \& Canteras, M. (2007). Effects of frequency of static stretching on straight-leg raise in elementary school children. Journal of Sports Medicine and Physical Fitness, 47(3), 304-308.

Shrout, P.E., \& Fleiss, J.L. (1979). Intraclass correlations: Uses in assessing rater reliability. Psychological Bulletin, $86(2), 420-428$.

Thacker, S.B., Gilchrist, J., Stroup, D.F., \& Kimsey, C.D.J. (2004). The impact of stretching on sports injury risk: A systematic review of the literature. Medicine and Science in Sports and Exercise, 36(3), 371-378.

Valentine, J., \& Cooper, H. (2003). Effect size substantive interpretation guidelines: Issues in the interpretation of effect sizes. Washington, DC.: Clearing House. 
Viciana, J., \& Mayorga-Vega, D. (2016). Innovative teaching units applied to physical education. Changing the curriculum management for authentic outcomes. Kinesiology, 48(1), 142-152.

Viciana, J., Mayorga-Vega, D., \& Merino-Marban, R. (2014). Physical education-based planning for developing and maintaining students' health-related physical fitness levels. In R. Todaro (Ed.), Handbook of physical education research. Role of school programs, children's attitudes and health implications (pp. 237-252). New York: Nova Science Publisher.

Submitted: April 21, 2015

Accepted: January 20, 2016

Correspondence to:

Daniel Mayorga-Vega, Ph.D.

FPU Research Fellow (AP2010-5905)

Department of Physical Education and Sport

University of Granada

Alfacar street, 18011, Granada, Spain

Phone: (0034) 958246641

Fax: (0034) 958244369

E-mail: dmayorgavega@gmail.com

\section{Acknowledgements}

We thank Anna Szczesniak for the English revision. Daniel Mayorga-Vega is supported by a research grant from the Spanish Ministry of Education, Culture and Sport (AP2010-5905). 